\title{
AVALIAÇÃO DO PESO CORPORAL E DA PRESSÃO ARTERIAL SISTÊMICA EM DOADORES RENAIS NO PERÍODO PÓS-OPERATÓRIO TARDIO
}

\author{
Assessment of Body Weight and Systemic Arterial Pressure of Kidney Donors in the Late \\ Postoperative Period
}

Daniel Xavier Lima', Andy Petroianu²

\begin{abstract}
RESUMO
Introdução: O transplante renal intervivos é um procedimento realizado com comprovada eficácia e segurança para os receptores, embora a evolução pós-operatória dos doadores seja pouco investigada. Objetivo: O objetivo do presente estudo foi avaliar doadores renais para transplantes intervivos no período pós-operatório tardio, analisando peso corporal e pressão arterial sistêmica. Métodos: Foram estudados 100 doadores (34 homens e 66 mulheres) de rim para transplantes, realizados no Hospital das Clínicas da Universidade Federal de Minas Gerais em um período superior a dois anos pós-operatórios. Resultados: Os índices de sobrepeso e obesidade foram superiores às médias brasileiras. A incidência de hipertensão arterial sistêmica foi semelhante à média brasileira, porém metade desses pacientes não conhecia seu nível pressórico. Conclusões: Houve obesidade e pressão arterial sistêmica elevada em incidência superior à esperada.
\end{abstract}

Descritores: transplante de rins, doadores vivos, hipertensão arterial, obesidade, sobrepeso.

Trabalho realizado no Hospital das Clínicas da Universidade Federal de Minas Gerais - Av. Alfredo Balena, 110 - CEP 30.130-100.

Mestre em Medicina - Urologista do Serviço de Nefrologia e Urologia do Hospital das Clínicas da UFMG

2. Livre-docente - Professor Titular do Departamento de Cirurgia da Faculdade de Medicina da UFMG

Endereço para correspondência: Prof. Andy Petroianu - Avenida Afonso Pena, 1626 - apto. 1901 - Fone / FAX: (31) 3274-7744 - e-mail: petroian@medicina.ufmg.br

Recebido em: 16/05/05 Aceito em: 30/06/05

\section{INTRODUÇÃO}

No Brasil, a proporção entre os transplantes renais intervivos e de cadáveres aumentou nos últimos anos, perfazendo cerca de $50 \%$ dos casos em 2005, segundo a Associação Brasileira de Transplantes de Orgãos e Tecidos (ABTO). O transplante renal intervivos ocupa posição de destaque em praticamente todo o mundo e a tendência atual é de aumentar o número desses procedimentos, o que talvez seja a principal alternativa para a redução das filas de espera para transplante. ${ }^{1}$

Os dados da literatura indicam que a doação renal é um procedimento com baixa morbimortalidade nos dias atuais..$^{2,3,4,5}$ De acordo com os trabalhos citados anteriormente, a disfunção renal não parece ser problema para os doadores, assim como a hipertensão arterial sistêmica, desde que sejam excluídos os candidatos à doação com alterações clínicas detectadas no processo de seleção. A agressão física em uma pessoa previamente sadia em prol de outra enferma ainda tem causado polêmica. Alguns autores acreditam que não se sabe o suficiente sobre a saúde doador em longo prazo e propõem a realização de mais estudos. ${ }^{6}$

A maioria dos trabalhos que avaliam a evolução pós-operatória dos doadores renais revela índices de hipertensão arterial sistêmica semelhantes aos da população geral., Existe, entretanto, o risco de disfunção no rim remanescente nos pacientes hipertensos, o que torna esses pacientes mais vulneráveis aos efeitos deletérios da hipertensão arterial. ${ }^{8}$

Outro aspecto relacionado à doação renal ainda pouco investigado é a alteração de hábitos de vida após a nefrectomia. Várias equipes transplantadoras recomendam aos doadores evitarem a prática de 
esportes que possam resultar em trauma ao rim remanescente. Não encontramos na literatura estudos sobre a alteração na prática de atividades físicas após a doação renal, nem sobre possível obesidade resultante de mudança de hábitos.

Os objetivos do presente estudo foram avaliar doadores de rim para transplante, no período pós-operatório tardio, com relação à presença de hipertensão arterial sistêmica e obesidade.

\section{MÉTODOS}

A presente pesquisa estudou 100 doadores renais para transplantes intervivos, realizados no Hospital das Clínicas da Universidade Federal de Minas Gerais em um período superior a dois anos pósoperatórios, com média de $8 \pm 5$ anos.

Os pacientes foram identificados por meio de registros hospitalares, sendo incluídos aleatoriamente apenas aqueles com mais de dois anos de doação. Foi feito contato com eles por telefone e nessa oportunidade foram explicados os objetivos da pesquisa. Os 100 primeiros doadores que aceitaram participar do estudo foram examinados pelo médico responsável pela pesquisa, no consultório do Serviço de Nefrologia e Urologia do Hospital das Clínicas da Universidade Federal de Minas Gerais (SENUR-HC-UFMG).

Durante a consulta, após a assinatura do termo de consentimento informado, os pacientes foram submetidos a anamnese dirigida, que teve como objetivo o registro de informações sobre o uso de anti-hipertensivos e sobre alterações de peso corporal após a doação renal.

Por último, os paciente foram submetidos a exame físico. Foram medidos e registrados o peso em quilogramas e a altura em centímetros, para classificar os pacientes, de acordo com o índice de massa corporal (IMC) nas categorias peso normal, sobrepeso e obesidade. O IMC foi obtido pela fórmula peso dividido pelo quadrado da altura, sendo o peso normal considerado como IMC menor do que $25 \mathrm{~kg} / \mathrm{m}^{2}$, sobrepeso considerado como IMC igual ou superior a $25 \mathrm{~kg} / \mathrm{m}^{2}$ e menor do que $30 \mathrm{~kg} / \mathrm{m}^{2}$. A obesidade foi estabelecida como IMC igual ou superior a $30 \mathrm{~kg} / \mathrm{m}^{2,9}$

A pressão arterial sistêmica foi medida com o auxílio de esfingmomanômetro de mercúrio, com o paciente em posição supina. Nos casos em que a pressão arterial sistólica era superior a $140 \mathrm{~mm} \mathrm{Hg}$ ou a pressão arterial diastólica era superior a $90 \mathrm{~mm}$ $\mathrm{Hg}$, realizava-se nova medida após dez minutos para confirmar o achado. Nos casos em que os níveis pressóricos da segunda medida permaneceram elevados, os pacientes eram considerados como portadores de pressão arterial sistêmica elevada e encaminhados ao cardiologista.

Trabalho aprovado pelo CEP e CONEP.

\section{RESULTADOS}

Foram encontrados elevados índices de sobrepeso e obesidade entre os doadores renais, tanto no sexo masculino, quanto no sexo feminino (Tabela 1 e Figura 1). Houve tendência a ganho de peso corporal após a doação, segundo os próprios doadores, sendo que apenas no sexo feminino houve quatro casos de perda de peso (Tabela 2).

De acordo com o relato dos doadores, $14 \%$ faziam uso de medicação anti-hipertensiva. Ao exame físico, 24\% apresentavam pressão arterial sistêmica igual ou maior a 140 x $90 \mathrm{mmHg}$. Considerando também os doadores que usavam anti-hipertensivos, o índice encontrado de pressão arterial sistêmica elevada foi de $31 \%$.
Observou-se que $17 \%$ dos doadores com pressão arterial sistêmica elevada não faziam uso de medicamentos anti-hipertensivos, por desconhecerem sua condição pressórica.

Tabela 1.

Análise descritiva do índice de massa corporal dos doadores renais

\begin{tabular}{cccc}
\hline IMC (kg/m2) & Homens & Mulheres & Total \\
\hline Menor que 20 & 4 & 6 & 10 \\
20 a 24 & 10 & 26 & 36 \\
25 a 29 & 14 & 20 & 34 \\
30 a 35 & 5 & 11 & 16 \\
Maior que 35 & 1 & 3 & 4 \\
\hline Total & $\mathbf{3 4}$ & $\mathbf{6 6}$ & $\mathbf{1 0 0}$ \\
\hline
\end{tabular}

IMC: Índice de massa corporal

$\mathrm{Kg} / \mathrm{m}^{2}$ : quilogramas por metro quadrado

Tabela 2.

Análise descritiva do peso após o transplante relatado pelos doadores

\begin{tabular}{cccccc}
\hline & $\begin{array}{c}\text { Ganhou } \\
\text { peso }\end{array}$ & $\begin{array}{c}\text { Perdeu } \\
\text { peso }\end{array}$ & $\begin{array}{c}\text { Peso } \\
\text { mantido }\end{array}$ & $\begin{array}{c}\text { Não } \\
\text { sabe }\end{array}$ & Total \\
\hline Homens & $16(47 \%)$ & 0 & $17(50 \%)$ & $1(3 \%)$ & $34(100 \%)$ \\
Mulheres & $\begin{array}{c}32 \\
(48,5 \%)\end{array}$ & $4(6 \%)$ & $29(44 \%)$ & $\begin{array}{c}1 \\
(1,5 \%)\end{array}$ & $66(100 \%)$ \\
Total & $48(48 \%)$ & $4(4 \%)$ & $46(46 \%)$ & $2(2 \%)$ & $100(100 \%)$ \\
\hline Mediana $(\mathrm{kg})$ & 7 & 12,5 & - & - & - \\
\hline
\end{tabular}

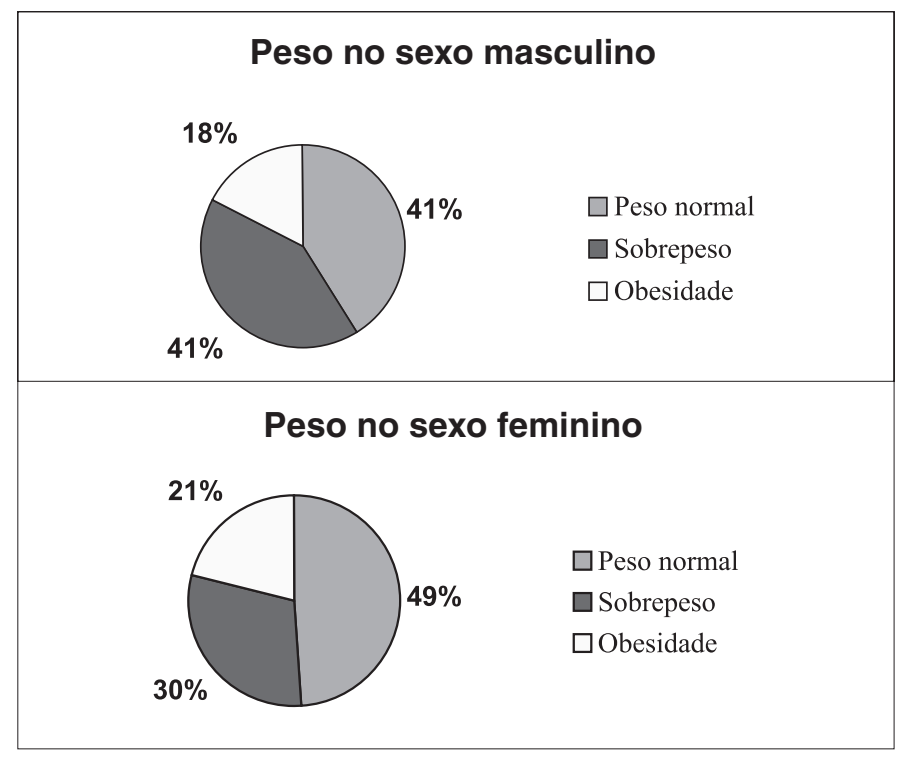

Figura 1 - Peso nos doadores renais, de acordo com o sexo 


\section{DISCUSSÃO}

A realização desta pesquisa justifica-se pela carência de informações sobre este tema em uma época de crescente desenvolvimento e realização de transplantes intervivos, não apenas para rim, mas também para medula óssea e fígado.

A freqüência de doadores com peso acima do limite ideal foi superior à média dos adultos brasileiros. ${ }^{9}$ Esses achados são relevantes, tendo em vista que a prevalência da obesidade no Brasil é elevada (sobrepeso: 28,3 \%; obesidade: 9,7 \%). ${ }^{9}$ Pessoas obesas têm maior probabilidade de desenvolver diversas doenças, como hipertensão arterial sistêmica, diabetes melito, coronariopatias e certas formas de câncer ${ }^{10}$. Existe a possibilidade de haver mais complicações no rim remanescente dos doadores com sobrepeso e obesidade, principalmente por causa da hipertensão arterial e do diabetes melito. As mudanças na imagem corporal causadas pelo excesso de gordura também podem reduzir a auto-estima e contribuir para uma percepção negativa da doação.

A nefrectomia por lombotomia, com ressecção de costela, acompanhase do menor risco para o doador e foi realizada em todos os pacientes do presente estudo ${ }^{11}$. É possível que a limitação para atividades físicas no período pós-operatório tenha contribuído para o sedentarismo e o conseqüente ganho de peso. Cerca da metade dos doadores relatou ganho de peso após a nefrectomia, o que sugere provável correspondência entre os dois eventos. Tais resultados, porém, devem ser analisados com cautela, uma vez que o índice de massa corporal desses pacientes à época da doação não é conhecido, assim como possíveis mudanças nos hábitos de vida.

Com relação à pressão arterial sistêmica, um terço dos doadores foram considerados portadores de pressão arterial sistêmica elevada, seja por relato de uso de anti-hipertensivos ou por apresentarem valores de pressão arterial iguais ou maiores que 140 x $90 \mathrm{mmHg}$ durante a consulta. Metade desses pacientes não fazia uso de anti-hipertensivos e não sabia seu estado pressórico. Esse resultado gera preocupação, uma vez que os doadores podem ser considerados mais vulneráveis aos efeitos deletérios da hipertensão arterial sistêmica, ao menos no sistema urinário.

$\mathrm{Na}$ ausência de tratamento adequado, há o risco de nefropatia hipertensiva no rim remanescente. É possível que alguns desses pacientes não sejam realmente hipertensos, uma vez que o diagnóstico de hipertensão arterial sistêmica exige medidas de pressão arterial sistêmica elevada em três ocasiões diferentes. Entretanto, mesmo com a utilização de critério com alta sensibilidade e especificidade abaixo do ideal, a incidência encontrada de pressão arterial sistêmica elevada não difere daquela presente na população urbana adulta brasileira, que varia entre $22,3 \%$ e $43,9 \% .^{12,13,14,15,16}$

Tais resultados estão também de acordo com trabalhos feitos em outros países, que mostram a freqüência da hipertensão arterial sistêmica em doadores renais como sendo a mesma da população geral. ${ }^{3,7,8}$ Contudo, essa comparação talvez não seja a forma mais adequada de avaliar a hipertensão arterial nos doadores renais, pois os candidatos à doação de rim submetem-se a exames para excluir os hipertensos. Portanto, o surgimento dessa doença após a doação requer investigação para determinar o papel da nefrectomia unilateral na gênese da hipertensão arterial sistêmica.

A presença de hipertensão arterial sistêmica e de obesidade não são fatores de exclusão para candidatos a doadores renais para transplantes intervivos no SENUR-HC-UFMG. Na avaliação pré-transplante, são excluídos os doadores com disfunção renal evidenciada pelo exame de urina ou pelo índice de depuração de creatinina e aqueles com hipertensão arterial mal controlada. Sendo o SENUR-HC-UFMG referência para diversos centros de diálise, há heterogeneidade nos critérios de seleção dos doadores encaminhados para transplante renal. Portanto, é possível que alguns doadores tenham sido obesos ou hipertensos na época da doação.

O impacto dessas condições na saúde dos doadores a longo prazo precisa ser melhor avaliada. Aceitarem-se doadores em condições clínicas limítrofes é justificável no caso de doadores em morte encefálica, como alternativa à escassez de órgãos para transplante. ${ }^{17}$ Todavia, no caso de doação renal em vida, a utilização de critérios de exclusão pouco rigorosos contraria o princípio de preservação da saúde dos doadores de órgãos para transplante.

\section{CONCLUSÕES}

- Houve prevalência de obesidade e sobrepeso entre os doadores renais superior à média encontrada no Brasil, no período pósoperatório tardio.

- Observou-se pressão arterial sistêmica elevada não diagnosticada entre os doadores renais, no período pós-operatório tardio.

\section{ABSTRACT}

Introduction: Live donor kidney transplantation is a procedure that has been proven to be effective and safe conducted to all receptors, although the postoperative outcome of donors has been subjected only to few investigation. Objective: The aim of this study was to assess the body weight and the systemic arterial pressure in kidney transplant donors in the late postoperative period. Methods: This study assessed 100 donors (34 men and 66 women) submitted to kidney transplant conducted at Hospital de Clínicas da Universidade Federal de Minas Gerais in a higher than two years postoperative period. Results: The overweight and the obesity rates were higher than the overall Brazilian population averages. The arterial hypertension frequency presented no difference compared to the overall Brazilian population average, although half of patients did not previously know their blood pressure levels. Conclusions: The obesity and systemic arterial hypertension prevalence was above expectations.

Keywords: kidney transplantation, live donors, arterial hypertension, obesity, overweight. 


\section{REFERÊNCIAS}

1. Cohen B, Persijn GG. Trends in organ donation. Transplant Proc. 1997;29:3301-02.

2. Bay WH, Hebert LA. The living donor in kidney transplantation. Ann Intern Med 1987;106:719-27.

3. Najarian SN, Chavers BM, McHugh LE, Natas AJ. 20 years or more of follow-up of living kidney donors. Lancet. 1992;340,:807-10.

4. Bia MJ, Ramos EL, Danovitch GM, Gaston RS, Harmon WE, Leichtman AB, et al Evaluation of living renal donors. Transplantation. 1995;60:322-7.

5. Johnson, EM, Remucal MJ, Gillingham KJ, Dahns RA, Najarian JS, Matas AJ. Complications and risks of living donor nephrectomy. Transplantation. 1997;64:1124-8

6. Ellison MD, McBride MA, Taranto SE, Delmonico FL, Francis L, Kauffman HM. Living kidney donors in need of kidney transplants: a report from the organ procurement and transplantation network. Transplantation. 2002;74:1349-51.

7. Goldfarb DA, Matin SF, Braun WE, Schreiber MJ, Mastroianni B, Papajcik D, et al. Renal outcome 25 years after donor nephrectomy. J Urol. 2001;166:2043-47.

8. Fehrman-Ekholm I, Duner F, Brink B, Tyden G, Elinder CF. No evidence of accelerated loss of kidney function in living kidney donors: results from a crosssectional follow-up. Transplantation. 2001;72:444-9.

9. Abrantes MM, Lamounier JA, Colosimo EA. Prevalência de sobrepeso e obesidade nas regiões nordeste e sudeste do Brasil. Rev Assoc Med Bras. 2003;49:162-6.

10. Kopelman PG. Obesity as a medical problem. Nature 2000;404:635-43.

11. Taghavi R. The complications and morbidity of flank incision for living renal donor. Transplant Proc. 2001;33:2638-9.

12. Lolio CA. Prevalência da hipertensão arterial em Araraquara. Arq Bras Cardiol 1990;55:167-73.

13. Ayres JEM. Prevalência da hipertensão arterial na cidade de Piracicaba. Arq Bras Cardiol. 1997;57:33-6.

14. Fuchs FD, Moreira LB, Moraes RS, Bredemeier M, Cardozo SC. Prevalência de hipertensão arterial sistêmica e fatores associados na região urbana de Porto Alegre. Estudo de base populacional. Arq Bras Cardiol. 1995;63:473-79.

15. Martins IS, Marucci MFN, Velásquez-Meléndez G, Coelho LT, Cervato AM Doenças cardiovasculares ateroscleróticas, dislipidemias, hipertensão, obesidade e diabetes melito em população da área metropolitana da região sudeste do Brasil. III- Hipertensão. Rev Saúde Pública. 1997;31:466-71.

16. Freitas OC, Carvalho FR, Neves JM, Veludo PK, Parreira RS, Gonçalves RM et al. Prevalência da hipertensão arterial sistêmica na população urbana de Catanduva, SP. Arq Bras Cardiol. 2001;77:9-15.

17. Rizvi SAH, Naqvi SAA. Our vision on organ donation in developing countries. Transplant Proc. 2000;32:144-5. 